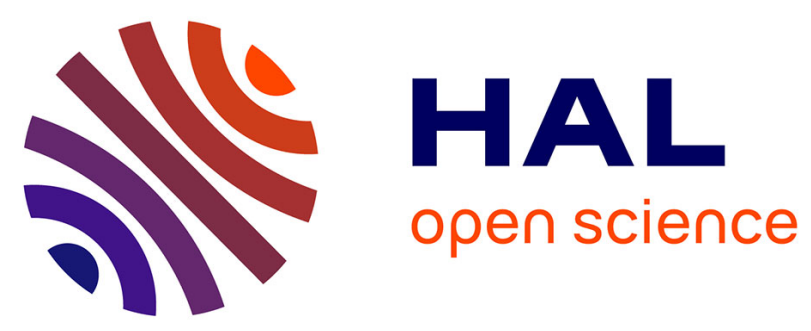

\title{
Joint estimation of local variance and local regularity for texture segmentation. Application to multiphase flow characterization.
}

Barbara Pascal, Nelly Pustelnik, Patrice Abry, Marion Serres, Valérie Vidal

\section{- To cite this version:}

Barbara Pascal, Nelly Pustelnik, Patrice Abry, Marion Serres, Valérie Vidal. Joint estimation of local variance and local regularity for texture segmentation. Application to multiphase flow characterization.. International Conference on Image Processing (ICIP) 2018, Oct 2018, Athens, Greece. hal-01818082v2

\section{HAL Id: hal-01818082 \\ https://hal.science/hal-01818082v2}

Submitted on 20 Jul 2018

HAL is a multi-disciplinary open access archive for the deposit and dissemination of scientific research documents, whether they are published or not. The documents may come from teaching and research institutions in France or abroad, or from public or private research centers.
L'archive ouverte pluridisciplinaire HAL, est destinée au dépôt et à la diffusion de documents scientifiques de niveau recherche, publiés ou non, émanant des établissements d'enseignement et de recherche français ou étrangers, des laboratoires publics ou privés. 


\title{
JOINT ESTIMATION OF LOCAL VARIANCE AND LOCAL REGULARITY FOR TEXTURE SEGMENTATION. APPLICATION TO MULTIPHASE FLOW CHARACTERIZATION.
}

\author{
Barbara Pascal ${ }^{1}$, Nelly Pustelnik ${ }^{1}$, Patrice Abry ${ }^{1}$, Marion Serres $^{1,2}$, Valérie Vidal ${ }^{1}$ \\ ${ }^{1}$ Univ Lyon, ENS de Lyon, Univ Lyon 1, CNRS, Laboratoire de Physique, F-69342 Lyon, France \\ ${ }^{2}$ IFP Énergies Nouvelles, Rond-point de l'échangeur de Solaize, BP3, 69360 Solaize, France
}

\begin{abstract}
Texture segmentation constitutes a task of utmost importance in statistical image processing. Focusing on the broad class of monofractal textures characterized by piecewise constancy of the statistics of their multiscale representations, recently shown to be versatile enough for real-world texture modeling, the present work renews this recurrent topic by proposing an original approach enrolling jointly scale-free and local variance descriptors into a convex, but non smooth, minimization strategy. The performance of the proposed joint approach are compared against disjoint strategies working independently on scale-free features and on local variance on synthetic piecewise monofractal textures. Performance are also compared for multiphase flow image characterization, a topic of crucial importance in geophysics as well as in industrial processes. Applied to large-size images (above two million pixels), the proposed approach is shown to significantly improve state-of-the-art strategies by permitting the detection of the smallest gas bubbles and by offering a better understanding of multiphase flow structures.
\end{abstract}

Index Terms - Total Variation, Primal-dual proximal algorithm, Texture segmentation, Strong convexity, Multiphase flow.

\section{INTRODUCTION}

Texture characterization - A texture is a perceptual attribute whose characterization has been envisaged with various mathematical models involving geometric or statistical attributes such as e.g., local variance or local spectral histograms. Amongst state-of-the-art texture segmentation techniques, one can refer to Yuan et al. [1] relying on Gabor coefficients as features followed by a selection procedure based on a matrix factorization step or to the multiscale contour detection procedure whose descriptors are the concatenation of brightness, color and texture (using textons) information followed by the computation of an oriented watershed transform [2]. In the present work, particular attention is granted to another class of multiscale techniques relying on scale-free local features [3], well-suited for multiphase flow texture modeling.

Challenges in multiphase flow characterization - Understanding and predicting the dynamics of multiphase flows is a major issue in geosciences (soil decontamination, $\mathrm{CO}_{2}$ sequestration) and in the industry (enhanced oil recovery, catalytic reactions) [4, 5]. Such phenomena all involve a joint gas and liquid flow inside a porous medium. Quantifying the contact areas between the different phases, where chemical reactions take place, is of tremendous importance for analyzing and predicting the efficiency of such processes [6]. However, the porous medium produces a global, multiscale texture

Work supported by Defi Imag'in SIROCCO and by ANR-16-CE33-0020 MultiFracs, France. which makes it difficult to extract gas-liquid interfaces. Segmentation techniques based on morphological tools used so far to differentiate phases in multiphase flows [7] present severe limitations: arbitrary threshold setting, non-physical irregular bubble contour, non detection of small bubbles. In addition, recent development in highresolution imaging yield large-size images thus bringing forward issues in memory and computational costs.

Model - In the present study, we consider a broad class of textured images $x=\left(x_{\underline{n}}\right)_{\underline{n} \in \Omega} \in \mathbb{R}^{|\Omega|}$ of size $|\Omega|=N_{1} \times N_{2}$, piecewise monofractal textures, characterized by piecewise constant statistical properties of their multiscale coefficients $\left(\mathcal{X}_{\underline{n}}^{(j)}\right)_{\underline{n} \in \Omega, j \in \mathbb{N}^{*}}$, where $2^{j}$ denotes the scale. These multiscale coefficients consist of either the absolute value of wavelet coefficients or wavelet leaders of texture $x$ [3], and behave locally as

$$
\mathcal{X}_{\underline{n}}^{(j)} \simeq s_{\underline{n}} 2^{j h_{\underline{n}}} \quad \text { when } \quad 2^{j} \rightarrow 0
$$

where $s \in \mathbb{R}^{|\Omega|}$, the local variance, and $h \in \mathbb{R}^{|\Omega|}$, the local regularity, are assumed to be piecewise constant fields.

To model multiphase flow textures, it is not assumed a priori that both $s$ and $h$ have edges at same locations. In this context, performing texture segmentation thus consists in estimating sharp changes in $s$ and $h$ from image $x$.

State-of-the-art - Model (1) is of common use for modeling scalefree textures [8] and has proven its efficiency to caracterized homogeneous textures as encountered in art investigation [9] or medical imaging [10], i.e. $s_{\underline{n}} \equiv s_{0}$ and $h_{\underline{n}} \equiv h_{0}$. The common approach to estimate $s$ and $h$ naturally involves the use of (weighted) linear regression across scales as

$$
\log _{2} \mathcal{X}_{\underline{n}}^{(j)} \simeq \log _{2} s_{\underline{n}}+j h_{\underline{n}} \quad \text { as } \quad 2^{j} \rightarrow 0 .
$$

When homogeneous texture are analysed, the multiscale coefficients are first averaged across space and then linear regressions on those averages lead to accurate estimates of $h_{0}$ and $s_{0}$, notably using wavelet leaders [3]. However, when the objective consists in obtaining local estimates to capture changes in $h$ or $s$, other strategies need to be deployed. In [8], estimation relies on a two-step procedure (as often in texture segmentation) consisting in (i) estimating $\widehat{h}_{\mathrm{Reg}}$ by linear regression across scales from $\mathcal{X}_{\underline{n}}^{(j)}$ and (ii) obtaining from

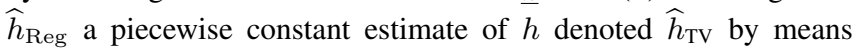
of total-variation denoising. In [8], we also proposed to combine both steps by incorporating the regression weight estimation into the optimization process leading to:

$$
\underset{h, w}{\operatorname{minimize}} \sum_{\underline{n} \in \Omega}\left(\sum_{j} w_{\underline{n}}^{(j)} \log _{2} \mathcal{X}_{\underline{n}}^{(j)}-h_{\underline{n}}\right)^{2}+\lambda \Omega(h, w)
$$

where $\lambda>0$ denotes a regularization parameter and $\Omega$ a convex regularization term inducing flexibility in the choice of the weights 
(w.r.t unbiased estimator) and where $h$ is assumed to be piecewise constant. This procedure lead to satisfactory estimation performance at the price though of high computational and memory costs.

Contribution - In this work, we propose a new estimation/segmentation procedure that perform jointly the estimation of $h$ of $s$. The proposed method aims (i) to estimate simultaneously the local variance and the local regularity, (ii) to rely on a fast algorithmic procedure and (iii) to involve a limited amount of memory. In Section 2, we formulate joint estimation as an optimization problem. The algorithmic solution devised to solve the optimization problem is presented in Section 3. Performance of the proposed strategies are assessed and compared to disjoint estimation procedure on synthetic piecewise constant monofractal textures in Section 4. Results achieved on multiphase flow images are detailed in Section 5 and compared to those obtained from state-of-the-art segmentation methods.

\section{SEGMENTATION AS AN OPTIMIZATION PROCEDURE}

We adopt a variational approach to estimate jointly $s$ and $h$ from the multiresolution quantity $\mathcal{X}$ :

$$
\underset{s \in \mathbb{R}^{|\Omega|}, h \in \mathbb{R}^{|\Omega|}}{\operatorname{minimize}} F(s, h ; \mathcal{X})+G(s, h) .
$$

where $\left.\left.F(\cdot, \cdot ; \mathcal{X}): \mathbb{R}^{|\Omega|} \times \mathbb{R}^{|\Omega|} \rightarrow\right]-\infty,+\infty\right]$ is a data-fidelity term reminiscent of (2) and $\left.\left.G: \mathbb{R}^{|\Omega|} \times \mathbb{R}^{|\Omega|} \rightarrow\right]-\infty,+\infty\right]$ is a penalization favoring piecewise constant estimates, based on the use of total variation. From (2), a natural choice for the data-fidelity term $F$ reads:

$$
F(s, h ; \mathcal{X})=\frac{1}{2} \sum_{j=J_{1}}^{J_{2}}\left\|\log _{2} \mathcal{X}^{(j)}-\log _{2} s-j h\right\|_{2}^{2},
$$

where $1 \leq J_{1}<J_{2}$. To manipulate convex data-term, we set $v=\log _{2} s$ and deal with the minimization problem over $(v, h)$ rather than over $(s, h)$ leading to

$$
(\widehat{v}, \widehat{h}) \in \underset{v, h}{\operatorname{argmin}} \sum_{j}\left\|\log _{2} \mathcal{X}^{(j)}-v-j h\right\|_{2}^{2}+\eta \operatorname{TV}(h)+\zeta \operatorname{TV}(v)
$$

with $\widehat{s}=2^{\widehat{v}}$, and where $\eta>0, \zeta>0$ are regularization parameters. TV models the isotropic total variation. Let $\mathbf{D}: \mathbb{R}^{|\Omega|} \rightarrow \mathbb{R}^{|\Omega| \times 2}$ computing the horizontal and vertical variations of intensity at each location $\underline{n}=\left(n_{1}, n_{2}\right) \in \Omega$,

$$
(\mathbf{D} y)_{n_{1}, n_{2}}=\left(\begin{array}{l}
y_{n_{1}, n_{2}+1}-y_{n_{1}, n_{2}} \\
y_{n_{1}+1, n_{2}}-y_{n_{1}, n_{2}}
\end{array}\right),
$$

the total variation is defined as, for every $y \in \mathbb{R}^{|\Omega|}, \operatorname{TV}(y)=$ $\|\mathbf{D} y\|_{2,1}$ where for every $z=\left(z_{1}, z_{2}\right) \in \mathbb{R}^{|\Omega| \times 2}$ :

$$
\|z\|_{2,1}=\sum_{n_{1}=1}^{N_{1}-1} \sum_{n_{2}=1}^{N_{2}-1} \sqrt{\left(z_{1}\right)_{n_{1}, n_{2}}^{2}+\left(z_{2}\right)_{n_{1}, n_{2}}^{2}} .
$$

\section{STRONG CONVEXITY AND FAST ALGORITHM}

\subsection{Primal-dual algorithms and strong convexity}

Problem (5) can be rewritten in a general form

$$
\widehat{y} \in \underset{y \in \mathcal{H}}{\operatorname{Argmin}} \varphi(y)+\psi(\mathbf{L} y)
$$

where $\varphi: \mathcal{H} \rightarrow \mathbb{R}$ and $\psi: \mathcal{G} \rightarrow \mathbb{R}$ are proper lower semicontinuous convex functions defined on Hilbert spaces $\mathcal{H}$ and $\mathcal{G}$, and
$\mathbf{L}: \mathcal{H} \rightarrow \mathcal{G}$ is a bounded linear operator. When the proximal operators of functions $\varphi$ and $\psi$, defined $\operatorname{ascox}_{\varphi}(y)=\underset{\tilde{y}}{\operatorname{argmin}} \frac{1}{2} \| y-$ $\tilde{y} \|_{2}^{2}+\varphi(\tilde{y})$ (and similarly for $\psi$ ), have closed form expressions, this problem can be solved using Chambolle-Pock primal-dual algorithm [11], belonging to the class of proximal algorithms [12, 13] and particularly efficient when dealing with TV penalties.

However, when working with large size data, as e.g. in multiphase flows, the basic form of Chambolle-Pock primal-dual algorithm suffers from too high and hence redhibitory computational cost. To deal with high dimensionality problems, it is necessary to take advantage of additional properties of the functions $\varphi$ and $\psi$, such as strong convexity. A function $\varphi$ is said to be $\mu$-strongly convex, for a given $\mu>0$, when the function $y \mapsto \varphi(y)-\frac{\mu}{2}\|y\|_{2}^{2}$ is convex.

When $\varphi$ is $\mu$-strongly convex, it is proven in [11] that it is possible to design an accelerated algorithm, relying on adaptive stepsizes, to solve (8). This algorithm is detailed in Algorithm 1 where the sequence $\left(y^{[k]}\right)_{k \in \mathbb{N}}$ converges to the solution of (8).

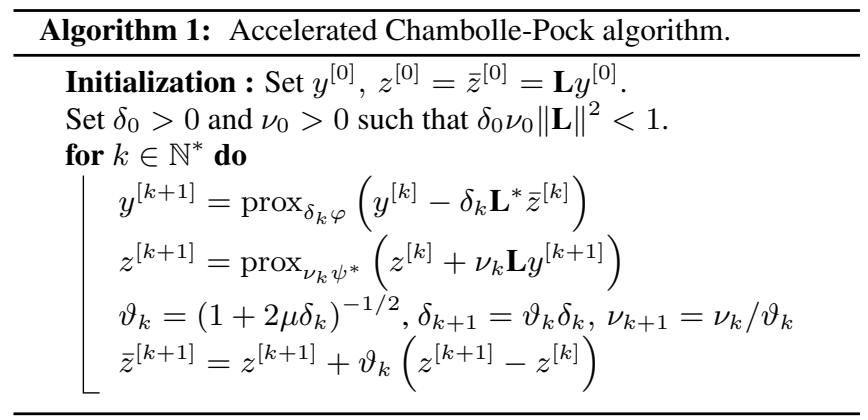

\subsection{Strong convexity of the data-fidelity term}

A differentiable function $\varphi$ is $\mu$-strongly convex if and only if

$$
(\forall(y, z) \in \mathcal{H} \times \mathcal{H}) \quad\langle\nabla \varphi(y)-\nabla \varphi(z), y-z\rangle \geq \mu\|y-z\|^{2}
$$

where $\langle.,$.$\rangle denotes the usual scalar product. For the data-fidelity$ term considered here that is, $\mathcal{H}=\mathbb{R}^{|\Omega|} \times \mathbb{R}^{|\Omega|}$ and $\forall y=(v, h) \in \mathcal{H}$,

$$
\varphi(y)=\widetilde{F}(v, h ; \mathcal{X})=\sum_{j=J_{1}}^{J_{2}}\left\|\log _{2} \mathcal{X}^{(j)}-v-j h\right\|_{2}^{2},
$$

one directly finds $\nabla \widetilde{F}(v, h ; \mathcal{X})=\mathbf{M}(v, h)^{\mathrm{T}}$, with $\mathbf{M}=\left(\begin{array}{ll}S_{0} \mathbb{I} & S_{1} \mathbb{I} \\ S_{1} \mathbb{I} & S_{2} \mathbb{I}\end{array}\right)$ is block matrix defined from

$$
S_{m}=\sum_{j=J_{1}}^{J_{2}} j^{m}
$$

and $\mathbb{I}$ is the identity matrix of $\mathbb{R}^{|\Omega|}$. Since the gradient $\nabla \widetilde{F}$ is linear, the condition for $\widetilde{F}$ to be $\mu$-strongly convex rewrites: $\forall(v, h) \in \mathcal{H}$,

$$
\langle\nabla \widetilde{F}(v, h ; \mathcal{X}),(v, h)\rangle=\left\langle\mathbf{M}(v, h)^{\mathrm{T}},(v, h)^{\mathrm{T}}\right\rangle \geq \mu\|(v, h)\|^{2}
$$

Moreover $\mathbf{M}$ is symmetric and positive definite, thus, denoting by $\beta$ its lowest eigenvalue, $\beta>0$ and

$$
\left\langle\mathbf{M}(v, h)^{\mathrm{T}},(v, h)^{\mathrm{T}}\right\rangle \geq \beta\left\langle(v, h)^{\mathrm{T}},(v, h)^{\mathrm{T}}\right\rangle,
$$

we conclude that the function $\widetilde{F}(v, h ; \mathcal{X})$ is $\mu$-strongly convex w.r.t the variables $(v, h)$, with $\mu=\beta$. 


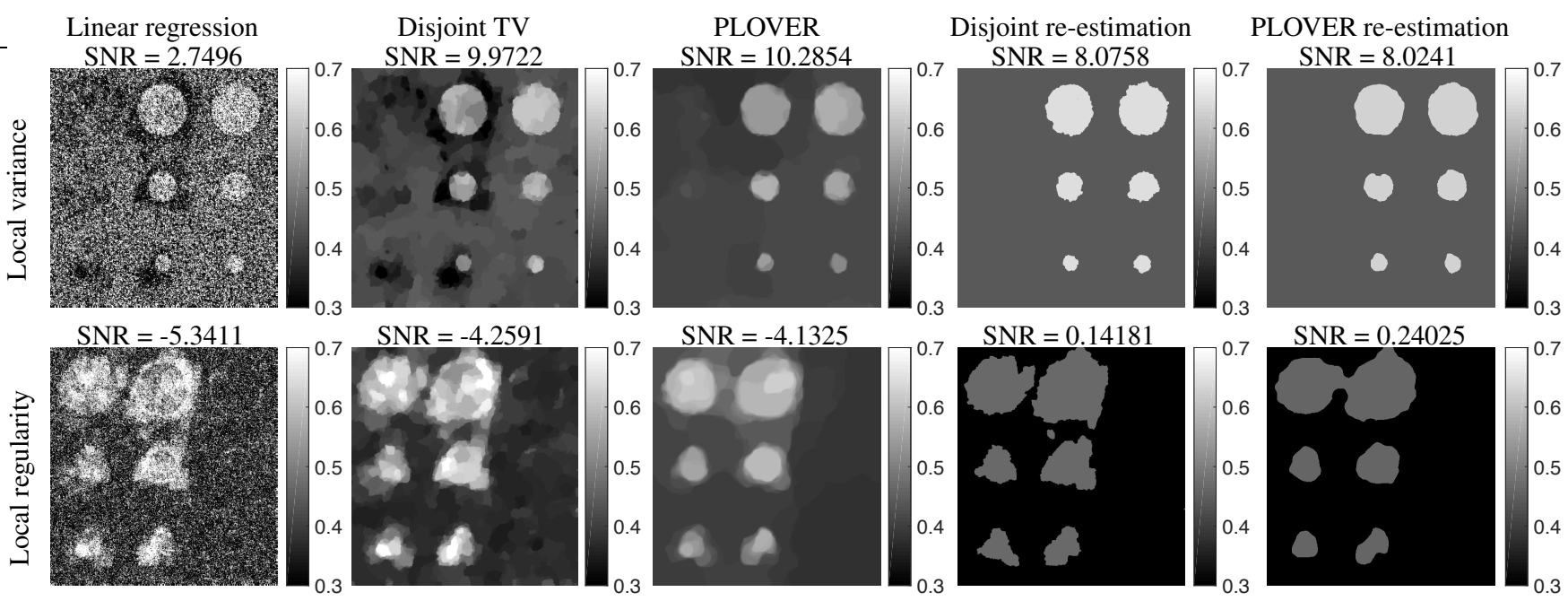

Fig. 1: Estimates for local variance (top) and regularity (bottom) : linear regressions (column 1), disjoint TV estimation (column 2), PLOVER (column 3). Average re-estimates for disjoint TV and PLOVER (columns 4 and 5 resp.).

\subsection{Proposed algorithm for texture segmentation}

Given the strong convexity result of the previous section, it is thus possible to use the general accelerated primal-dual Algorithm 1 for solving Problem (5). The iterations are customized to the minimization of (5) in Algorithm 2 setting $y=(v, h)$. The proximity operator of the data-fidelity term is provided in Proposition 1 while the proximal operator of the conjugate function of the mixed 2,1-norm is derived in [14]. The convergence of $\left(v^{[k]}, h^{[k]}\right)_{k \in \mathbb{N}}$ to a minimizer of Problem (5) is insured by $\delta_{0} \nu_{0}\|\mathbf{D}\|^{2}<1$.

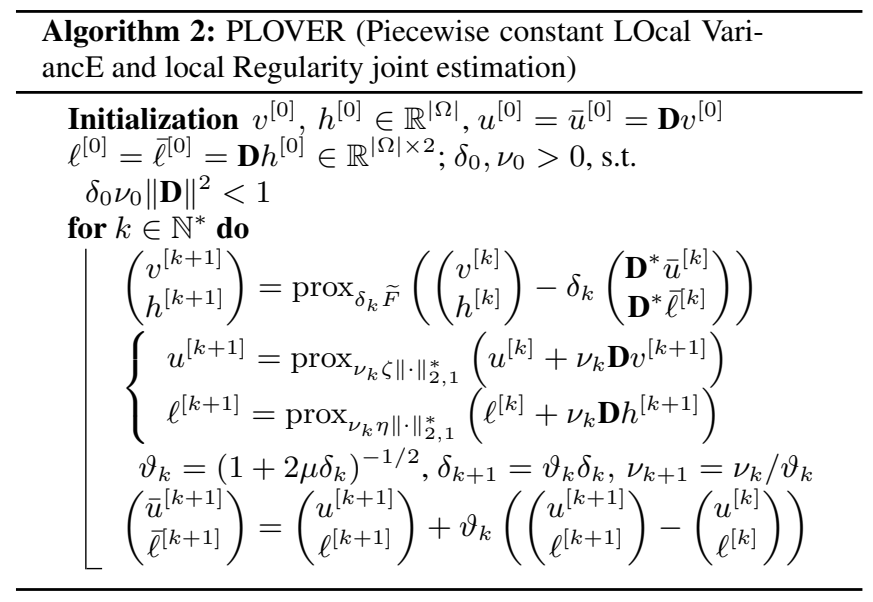

Proposition 1 (Computation of $\operatorname{prox}_{\widetilde{F}}$ ). Let $\widetilde{F}$ be defined as in (9). Let $\mathcal{S}=\sum_{j} \log _{2} \mathcal{X}^{(j)}, \mathcal{T}=\sum_{j}\left(j \log _{2} \mathcal{X}^{(j)}\right)$ and $\mathcal{N}=(1+$ $\left.S_{0}\right)\left(1+S_{2}\right)-S_{1}^{2},\left\{S_{m}\right\}_{m \in\{0,1,2\}}$ defined in (10). For every $(v, h) \in$ $\mathbb{R}^{|\Omega|} \times \mathbb{R}^{|\Omega|},(p, q)=\operatorname{prox}_{\widetilde{F}}(v, h)$ with

$$
\left\{\begin{array}{l}
p=\left(\left(1+S_{2}\right)(\mathcal{S}+v)-S_{1}(\mathcal{T}+h)\right) / \mathcal{N} \\
q=\left(\left(1+S_{0}\right)(\mathcal{T}+h)-S_{1}(\mathcal{S}+v)\right) / \mathcal{N}
\end{array}\right.
$$

\section{PERFORMANCE ASSESSMENT}

Simulation settings - To evaluate the performance of the proposed piecewise constant local variance and local regularity estimation PLOVER procedure (cf. Algorithm 2), we generate piecewise monofractal textures fully characterized by their (piecewise) local variance $\bar{s}$ and (piecewise) local regularity $\bar{h}$. An example is displayed in Fig. 2(a), while underlying masks for local variance and local regularity are represented on Figs. 2(b) and (c). Synthetic textures consist of three regions embedded in a background, characterized with $\bar{s}_{\underline{n}} \equiv 0.3$ and $\bar{h}_{\underline{n}} \equiv 0.1$ : Region 1(left) corresponds to a change in local regularity $\bar{h}$ (w.r.t. the background) but no change in local variance $s\left(\bar{s}_{\underline{n}} \equiv 0.3, \bar{h}_{\underline{n}} \equiv 0.6\right)$; Region 2 (middle) corresponds to changes in both local variance and regularity $\left(\bar{s}_{\underline{n}} \equiv 0.6, \bar{h}_{\underline{n}} \equiv 0.6\right)$; Region 3 (right) corresponds to changes in local variance only $\left(\bar{s}_{\underline{n}} \equiv 0.6, \bar{h}_{\underline{n}} \equiv 0.1\right)$. The masks consisting of three different size disks permit to test the impact of region sizes on performance. Wavelet leaders, local suprema of wavelet coefficients [3] are chosen as multiresolution quantities $\mathcal{X}$. Scales involved in the minimization range from $J_{1}=1$ to $J_{2}=5$.

Performance evaluation - Fig. 1 reports estimation performance compared i) when performing linear regressions equivalent to solve Problem (5) when $\eta=\zeta=0$ leading to $\left(\widehat{v}_{\mathrm{Reg}}, \widehat{h}_{\mathrm{Reg}}\right)$ and with $\widehat{s}_{\text {Reg }}=2^{\widehat{v}_{\text {Reg }}}$ (column 1); ii) when applying a simple TV denoising on $\widehat{v}_{\text {Reg }}$ and $\widehat{h}_{\text {Reg }}$ separately, i.e., solving the minimization problem $\widehat{y}_{\mathrm{TV}}=\operatorname{argmin} \frac{1}{2}\left\|y-\widehat{y}_{\mathrm{Reg}}\right\|_{2}^{2}+\lambda_{y} \mathrm{TV}(y)$ where $y=\{s, h\}$ (column 2) ; iii) when applying the proposed PLOVER procedure (column 3).

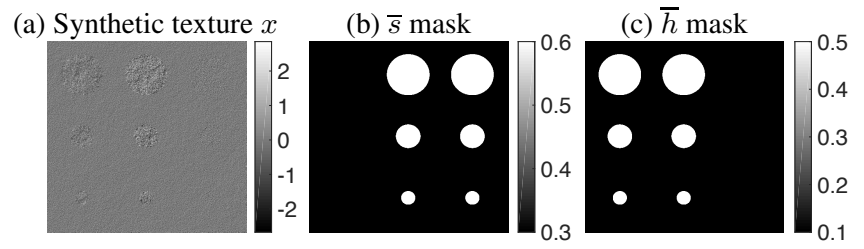

Fig. 2: (a) Synthetic piecewise monofractal textures using piecewise local variance (b) and regularity (c).

Because it is now well known that TV-based estimation suffers from large biases [15] that precludes the recovery of exact values for $\bar{s}$ and $\bar{h}$ in the different regions. To overcome this difficulty, we first perform a K-means segmentation from the estimates $\left(\widehat{s}_{\bullet}, \widehat{h}_{\bullet}\right)$ and re-estimate from the multiscale coefficients $\mathcal{X}$ the values of local variance and regularity for the segmented regions by global averages within segmented regions. Achieved performance are displayed in the fourth and fifth columns, $\lambda, \eta$ and $\zeta$ are chosen as those leading to the best SNR on these re-estimates. 
(a) Image $x \in \mathbb{R}^{|\Omega|}$

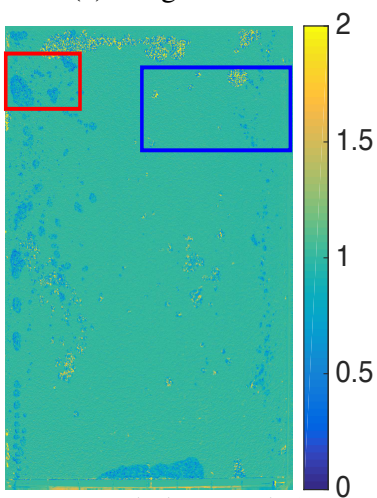

(e) Arbelaez et al [2]
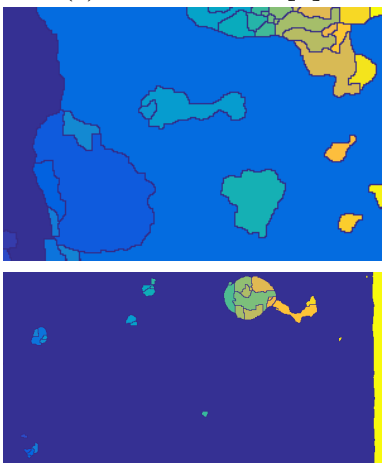

(b) Zoom of $x \in \mathbb{R}^{|\Omega|}$

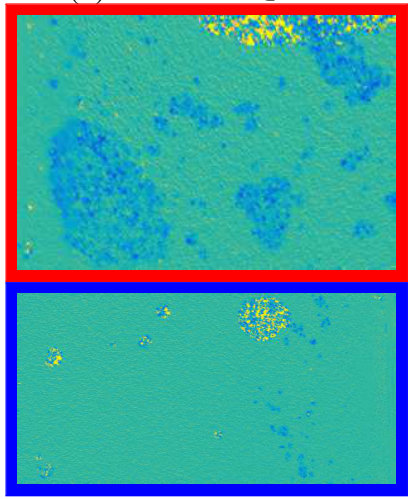

(f) Yuan et al [1]
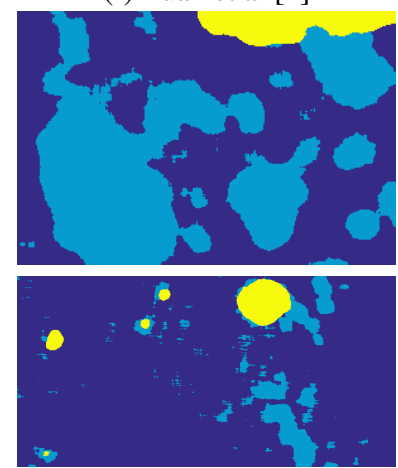

(c) PLOVER : $\widehat{s}$
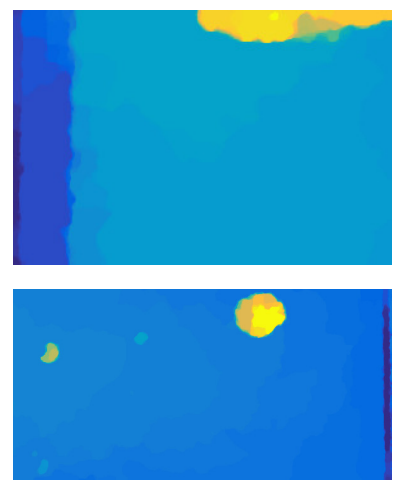

(g) Disjoint TV
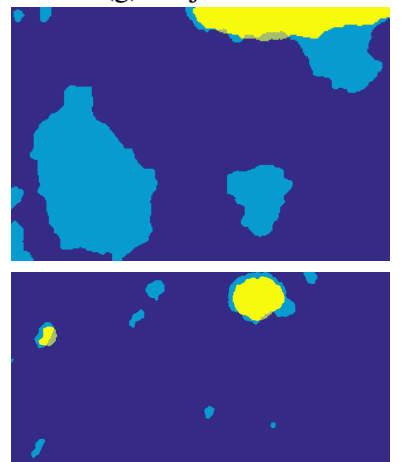

(d) PLOVER : $\widehat{h}$
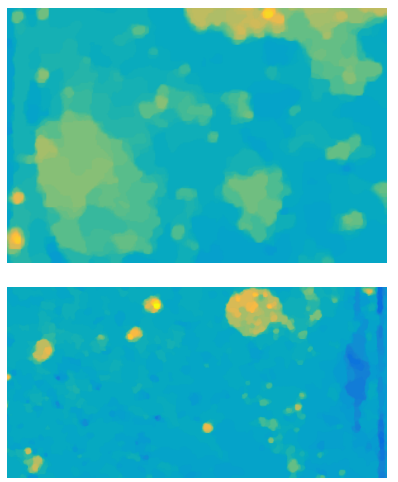

(h) PLOVER
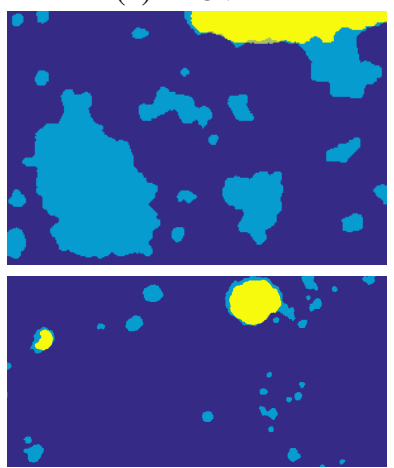

Fig. 3: Multiphase flow image (a) and zooms (b). PLOVER estimates for local variance (c) and regularity (d). State-of-the-art segmentations applied on $x(\mathrm{e})$ and (f), K-means based segmentation from disjoint estimates (g) and PLOVER (h).

Local regularity and variance estimation is clearly improved by the proposed joint estimation PLOVER procedure, compared to disjoint estimation. This is significantly the case for the local regularity estimates, a significant outcome as local regularity is a notoriously difficult quantity to estimate. Moreover, edges of estimates are found to be more regular for the proposed PLOVER procedure, an outcome of practical importance for multiphase flow analysis, where bubble perimeter estimation if of critical importance. For piecewise monofractal textures, it has been shown that texture segmentation state-of-the-art methods such as those in $[1,2]$ do not yield satisfactory results (cf. [8, Fig.3]), the corresponding comparisons not reported here for space reasons further confirm such findings.

\section{MULTIPHASE FLOW LARGE-SIZE IMAGE ANALYSIS}

Data acquisition - Experiments of joint gas and liquid flow through a porous medium were performed in a quasi-2D vertical cell (HeleShaw cell of width $210 \mathrm{~mm}$, height $410 \mathrm{~mm}$ and gap $2 \mathrm{~mm}$ ). The porous medium is an open cell solid foam of $\mathrm{NiCrFeAl}$ alloy, with a typical pore diameter of $580 \mu \mathrm{m}$. Constant gas and liquid flow rates are injected at the bottom of the cell through nine injectors (air) and a homogeneous slit (water). Images of the multiphase flow are acquired by a high-resolution camera (Basler, $2048 \times 2048$ pixels) at $100 \mathrm{~Hz}$ [16]. The size of the images to analyze is $1677 \times 1160$. An example is provided in Fig. 3(a), showing that the gas phase (blue or yellow structures) is textured because of the solid porous medium. The liquid phase (in green) is also textured though at smaller scales (cf. Fig. 3(b)).

Discussion - Figs. 3(c)-(d) clearly show that the local variance $\widehat{s}$ and local regularity $\widehat{h}$ provide information different in nature: $\widehat{h}$ captures well all gas bubbles and it can be conjectured that the value of $\widehat{h}$ is linked to bubble thickness variations ; $\widehat{s}$ brings forward bubbles located in the foreground only (yellow structures in Fig. 3(b)), thus providing rich information on the bubble distribution in the cell gap. Comparisons with state-of-art - Fig. 3 (2nd row) compares segmentation outcomes of the proposed PLOVER procedure to those obtained from state-of-the-art texture segmentation methods: Fig. 3(e) illustrates the segmentation obtained with the oriented watershed transform of Arbelaez et al [2] and Fig. 3(f) shows the segmentation obtained following Yuan et al [1] method based on matrix factorization. The results of disjoint TV estimation procedure [8] are displayed on Fig. 3(g). For disjoint TV and PLOVER results, the segmentation is obtained by performing K-means over $\widehat{h}_{\bullet}$ and $\widehat{s}_{\bullet}$. The proposed method PLOVER yields better determination of individual objects (bubbles), this is critically the case for the smaller size bubbles, of great importance in a forthcoming quantification of phase contact surfaces, critical features in multiphase flow characterization. In addition, PLOVER produces an enhanced separation of the foreground and background bubble populations, which yield an improved description of multiphase flows. Further, some state-of-the-art approaches are so demanding computational and memory-resource wise, that they could not be applied to the large-size images directly while the current PLOVER implementation permits a fast analysis of such large-size images. Results were hence compared for cropped versions of actual images.

\section{CONCLUSION}

These computational efficiency of PLOVER combined to the significant improvements in multiphase flow large-size image analysis pave the way towards a systematic use in this application. Analyses on much larger simulated and real-world image datasets are under current investigations together with the automated tuning of the hyper-parameters $\lambda, \eta$ and $\zeta$. 


\section{REFERENCES}

[1] J. Yuan, D. Wang, and A. M. Cheriyadat, "Factorization-based texture segmentation," IEEE Trans. Image Process., vol. 24, no. 11, pp. 3488-3497, Nov. 2015.

[2] P. Arbelaez, M. Maire, C. Fowlkes, and J. Malik, "Contour detection and hierarchical image segmentation," IEEE Trans. Pattern Anal. Match. Int., vol. 33, no. 5, pp. 898-916, 2011.

[3] H. Wendt, S. G. Roux, P. Abry, and S. Jaffard, "Wavelet leaders and bootstrap for multifractal analysis of images," Signal Proces., vol. 89, no. 6, pp. 1100-1114, 2009.

[4] K. R Reddy and J. A Adams, "Effects of soil heterogeneity on airflow patterns and hydrocarbon removal during in situ air sparging," Journal of Geotechnical and Geoenvironmental Engineering, vol. 127, no. 3, pp. 234-247, 2001.

[5] V. Hessel, P. Angeli, A. Gavriilidis, and H. Löwe, "Gasliquid and gas-liquid-solid microscructured reactors: Contacting principles and applications," Industrial \& engineering chemistry research, vol. 44, pp. 9750-9769, 2005.

[6] M. T. Kreutzer, F. Kapteijn, J. A. Moulijn, and J. J. Heiszwolf, "Multiphase monolith reactors: Chemical reaction engineering of segmented flow in microchannels," Chemical Engineering Science, vol. 60, pp. 5895-5916, 2005.

[7] M. Serres, M.-L. Zanota, R. Philippe, and V. Vidal, "On the stability of taylor bubbles inside a confined highly porous medium," International Journal of Multiphase Flow, vol. 85, pp. 157-163, 2016.

[8] N. Pustelnik, H. Wendt, P. Abry, and N. Dobigeon, "Combining local regularity estimation and total variation optimization for scale-free texture segmentation," IEEE Trans. Computational Imaging, vol. 2, no. 4, pp. 468-479, 2016.

[9] P. Abry, S. Jaffard, and H. Wendt, "When Van Gogh meets Mandelbrot: Multifractal classification of painting's texture," Signal Proces., vol. 93, no. 3, pp. 554-572, 2013.

[10] Z. Marin, K. A. Batchelder, B. C. Toner, L. Guimond, E. Gerasimova-Chechkina, A. R. Harrow, A. Arneodo, and A. Khalil, "Mammographic evidence of microenvironment changes in tumorous breasts," Medical physics, vol. 44, no. 4, pp. 1324-1336, 2017.

[11] A. Chambolle and T. Pock, "A first-order primal-dual algorithm for convex problems with applications to imaging," $J$. Math. Imag. Vis., vol. 40, no. 1, pp. 120-145, 2011.

[12] H. H. Bauschke and P. L. Combettes, Convex Analysis and Monotone Operator Theory in Hilbert Spaces, Springer, New York, 2011.

[13] P. L. Combettes and J.-C. Pesquet, "Proximal splitting methods in signal processing," in Fixed-Point Algorithms for Inverse Problems in Science and Engineering, H. H. Bauschke, R. S. Burachik, P. L. Combettes, V. Elser, D. R. Luke, and H. Wolkowicz, Eds., pp. 185-212. Springer-Verlag, New York, 2011.

[14] M. J. Fadili and G. Peyré, "Total variation projection with first order schemes," IEEE Trans. Image Process., vol. 20, no. 4, pp. $657-669,2010$.

[15] C.-A. Deledalle, N. Papadakis, J. Salmon, and S. Vaiter, "Clear: Covariant least-square refitting with applications to image restoration," SIAM Journal on Imaging Sciences, vol. 10, no. 1, pp. 243-284, 2017.
[16] M. Serres, "Etude hydrodynamique d'un écoulement gazliquide dans un milieu poreux confiné," PhDThesis, École Normale Supérieure de Lyon, Université de Lyon, pp. $\mathrm{x}+203,2017$. 